\title{
Relations Between NC and CC Neutrino Structure Functions for Nucleons and Nuclei
}

\author{
S. A. Kulagin ${ }^{\text {a }}$ \\ ${ }^{a}$ Institute for Nuclear Research, 117312 Moscow, Russia
}

The relations between neutrino NC and CC structure functions and cross sections, which are driven by isospin symmetry, are discussed for nucleons and nuclei.

\section{Introduction}

The studies of neutrino interactions with nuclei has been an actively developing field both experimentally and theoreticaly [12]. Nuclear physics studies with (anti)neutrino beams are relevant in two different contexts. First, it must be noted that neutrino collision experiments usually use heavy-nucleus targets. For this reason a good understanding of "standard" nuclear effects is necessary for interpretation of high precision experiments with neutrino beams. On the other hand, neutrino experiments can provide better understanding of physics of nuclei that is important and interesting per se 3 .

The interaction of (anti)neutrino with matter is mediated by charged $W^{+}$or $W^{-}$boson (charged current, CC), or by neutral $Z$ boson (neutral current, NC). For this reason the studies of neutrino interactions provide information which is not accessable with charged-lepton probes. For example, the neutrino $\mathrm{NC}$ interaction strength is determined by the weak mixing angle $\theta_{W}$ and the studies of relative rates of $\mathrm{NC}$ and $\mathrm{CC}$ neutrino reactions provide a tool for the measurement of $\sin ^{2} \theta_{W}$ [4.

In this paper we analyse relations between CC and NC neutrino structure functions for nucleons and nuclei. In Sect. 2 2 we discuss the derivation of $\mathrm{NC}-\mathrm{CC}$ relations for structure functions at high momentum transfer $Q$ for a generic target. First we discuss the structure functions in the QCD leading order in strong coupling constant (LO) in terms of parton distributions (PDFs) focusing on the analysis of contributions from differ- ent isospin states. We point out simple relations (see Eqs. (10) ) emerging in this approximation. Then we discuss perturbative QCD corrections to these relations. In Sect. 3 we discuss nuclear corrections to parton distributions with different isospin. The results are applied to compute the non-isoscalarity correction to the ratio $F_{3}^{Z} / F_{3}^{W}$ for iron.

\section{NC/CC ratios for neutrino scattering}

For an isoscalar target (e.g. the isoscalar combination of proton and neutron, or for deuterium) a relation between neutrino-antineutrino asymmetries in the NC and CC deep-inelastic (DIS) cross sections was derived long ago by Paschos and Wolfenstein [5]

$R^{-}=\frac{\sigma_{\mathrm{NC}}^{\nu}-\sigma_{\mathrm{NC}}^{\bar{\nu}}}{\sigma_{\mathrm{CC}}^{\nu}-\sigma_{\mathrm{CC}}^{\bar{\nu}}}=\frac{1}{2}-\sin ^{2} \theta_{W}$

where $\theta_{W}$ is the Weinberg mixing angle. The corresponding relation was also derived for the $C$ even combinations of cross sections [6] (LlewellynSmith relationship)

$R^{+}=\frac{\sigma_{\mathrm{NC}}^{\nu}+\sigma_{\mathrm{NC}}^{\bar{\nu}}}{\sigma_{\mathrm{CC}}^{\nu}+\sigma_{\mathrm{CC}}^{\bar{\nu}}}=\frac{1}{2}-\sin ^{2} \theta_{W}+\frac{10}{9} \sin ^{4} \theta_{W}$.

It should be remarked that in the derivation of the Paschos-Wolfenstein (PW) and the Llewellyn-Smith (LS) relationships the contributions from the $s$ and $c$ quarks were neglected. Furthermore, the derivation of the LS relationship holds for high momentum transfer $Q^{2}$ and this relationship should be corrected for perturbative and non-perturbative QCD effects even in 
an ideal world with only $u$ and $d$ quarks. The PW relationship is, however, more general. If only the contributions due to light quarks are taken into account, the PW relationship is a direct result of the isospin symmetry. This ensures that various strong interaction effects, including nuclear effects, cancel out in $R^{-}$for an isoscalar target thus making Eq.(11) a good tool for the measurement of the mixing angle in neutrino scattering.

If $s$ and $c$ quarks are taken into account the $C$ even ratio $R^{+}$involves contributions due to $s+\bar{s}$ and $c+\bar{c}$ distributions, while the $C$-odd $R^{-}$is corrected by $s-\bar{s}$ and $c-\bar{c}$ asymmetries in the target (for a discussion of possible asymmetry in the strange sea and the magnitude of this correction to $R^{-}$see 78910 ). It should be remarked that relations (11) and (2) are also violated by isospinviolating effects in PDFs (for a recent discussion of this effect see [11).

The targets used in neutrino experiments are usually heavy nuclei, such as iron in $\mathrm{NuTeV}$ experiment 4. Heavy nuclei typically have an excess of neutrons over protons and, therefore, are not isoscalar targets. For a non-isoscalar target relations (11) and (2) are violated by contributions due to isovector component of PDFs.

In this paper we consider the ratios $R^{ \pm}$for inclusive differential cross sections for a generic target as a function of Bjorken $x$ and the energy transfere in units of beam energy $E, y=q_{0} / E$. In terms of the structure functions $F_{2}, F_{3}$, and $F_{L}$ we have

$$
\begin{aligned}
R^{-} & =\frac{x F_{3}^{Z} Y_{-} / 2}{x F_{3}^{W} Y_{-}+\Delta F_{2}^{W} Y_{+}-\frac{1}{2} \Delta F_{L}^{W} y^{2}}, \\
R^{+} & =\frac{\left(F_{2}^{Z} Y_{+}+\frac{1}{2} F_{L}^{Z} y^{2}\right) / 2}{F_{2}^{W} Y_{+}+\frac{1}{2} F_{L}^{W} y^{2}+\Delta x F_{3}^{W} Y_{-}},
\end{aligned}
$$

where $Y_{ \pm}=\frac{1}{2}\left[1 \pm(1-y)^{2}\right]$, the superscript $Z$ and $W$ label the $\mathrm{NC}$ and $\mathrm{CC}$ neutrino structure functions, respectively, $F_{2}^{W}=\frac{1}{2}\left(F_{2}^{\nu}+F_{2}^{\bar{\nu}}\right)$ and $\Delta F_{2}^{W}=\frac{1}{2}\left(F_{2}^{\nu}-F_{2}^{\bar{\nu}}\right)$ and similar definitions for $F_{3}$ and the longitudinal structure function $F_{L}$ (in Eqs. (3) and (4) we neglect the factor $\left(1+Q^{2} / M_{W}^{2}\right)^{2} /\left(1+Q^{2} / M_{Z}^{2}\right)^{2}$ arising due to the ratio of propagators of $W$ and $Z$ whose effect is small for feasible $Q^{2}$ ).

\subsection{Relations between $\mathrm{CC}$ and NC struc- ture functions}

We now address relations between the $\mathrm{NC}$ and CC structure functions. We assume that $Q^{2}$ is high enough to apply the leading twist (LT) QCD approximation. In this approximation the $\mathrm{NC}$ and $\mathrm{CC}$ structure functions are given in terms of PDFs. In order to facilitate discussion of isospin effects, we consider the isoscalar, $q_{0}(x)=$ $u(x)+d(x)$, and the isovector, $q_{1}(x)=u(x)-d(x)$, quark distributions (for simplicity of notations, we suppress the explicit notation for the $Q^{2}$ dependence of parton distributions). We also introduce quark distributions with definite isospin $I=0,1$ and $C$ parity

$F^{(I, \pm)}(x)=x q_{I}(x) \pm x \bar{q}_{I}(x)$,

where $\bar{q}$ is antiquark distribution. We first consider the CC structure functions. In terms of the functions $F^{(I, C)}$ the structure functions can be written as follows (in QCD leading order)

$$
\begin{gathered}
F_{2}^{W}=F^{(0,+)}+F^{(s,+)}+F^{(c,+)}, \\
x F_{3}^{W}=F^{(0,-)}+F^{(s,-)}+F^{(c,-)}, \\
\Delta F_{2}^{W}=-F^{(1,-)}+F^{(s,-)}-F^{(c,-)}, \\
\Delta x F_{3}^{W}=-F^{(1,+)}+F^{(s,+)}-F^{(c,+)} .
\end{gathered}
$$

where for $C$-even and $C$-odd distributions of strange $(I=s)$ and charmed $(I=c)$ quarks we use notations similar to Eq. (5.5).

For the neutrino NC scattering the LO structure functions can be written as

$$
\begin{aligned}
F_{2}^{Z}= & C_{2}^{0} F^{(0,+)}+C_{2}^{1} F^{(1,+)}+ \\
& C_{2}^{s} F^{(s,+)}+C_{2}^{c} F^{(c,+)}, \\
x F_{3}^{Z}= & C_{3}^{0} F^{(0,-)}+C_{3}^{1} F^{(1,-)}+ \\
& C_{3}^{s} F^{(s,-)}+C_{3}^{c} F^{(c,-)} .
\end{aligned}
$$

The coeficients $C_{2}^{I}$ and $C_{3}^{I}$ in Eqs.(7) are

$$
\begin{aligned}
& C_{2}^{0}=1-2 \sin ^{2} \theta_{W}+\frac{20}{9} \sin ^{4} \theta_{W}, \\
& C_{2}^{1}=-\frac{2}{3} \sin ^{2} \theta_{W}\left(1-2 \sin ^{2} \theta_{W}\right), \\
& C_{2}^{s}=1-\frac{4}{3} \sin ^{2} \theta_{W}+\frac{8}{9} \sin ^{4} \theta_{W}, \\
& C_{2}^{c}=1-\frac{8}{3} \sin ^{2} \theta_{W}+\frac{32}{9} \sin ^{4} \theta_{W},
\end{aligned}
$$


$C_{3}^{0}=1-2 \sin ^{2} \theta_{W}$,

$C_{3}^{1}=-\frac{2}{3} \sin ^{2} \theta_{W}$,

$C_{3}^{s}=1-\frac{4}{3} \sin ^{2} \theta_{W}$,

$C_{3}^{c}=1-\frac{8}{3} \sin ^{2} \theta_{W}$.

Using equations (6) to (9) we arrive at the following relations between $\mathrm{CC}$ and $\mathrm{NC}$ structure functions

$$
\begin{aligned}
F_{2}^{Z} & =C_{2}^{0} F_{2}^{W}-C_{2}^{1} \Delta x F_{3}^{W}, \\
x F_{3}^{Z} & =C_{3}^{0} x F_{3}^{W}-C_{3}^{1} \Delta F_{2}^{W} .
\end{aligned}
$$

These relations have been derived in the $\mathrm{LO}$ approximation. It is important to study QCD corrections to these relations. This is most conveniently done in the DIS scheme 12, in which only $F_{L}$ and $F_{3}$ structure functions change. In this scheme perturbative corrections to Eqs. (10) are determined by the corresponding correction to the structure function $F_{3}$. Let us denote for each of the structure functions $i=2,3, L$ $F_{i}=F_{i}^{\mathrm{LO}}+\delta F_{i}$, where $F_{i}^{\mathrm{LO}}$ are the LO structure functions by Eqs.(6) and (7) and $\delta F_{i}$ are perturbative series in $\alpha_{S}$ which can be written as convolutions of coefficient functions with LO structure functions. For the structure function $F_{3}$

$\delta F_{3}=\int_{x}^{1} \frac{\mathrm{d} z}{z} K_{3}\left(z, \alpha_{S}\left(Q^{2}\right)\right) F_{3}^{\mathrm{LO}}\left(\frac{x}{z}, Q^{2}\right)$,

where $K_{3}$ is the corresponding coefficient function. In the DIS scheme $\delta F_{2}^{Z, W}=0$ to all order in perturbation theory. Therefore, the generalization of Eq.10a can be written as

$F_{2}^{Z}-C_{2}^{0} F_{2}^{W}=-C_{2}^{1} \Delta x F_{3}^{W(\mathrm{LO})}\left(x, Q^{2}\right)$,

where from the left we have the full $\left(\alpha_{S}\right.$-corrected) structure functions and from the right the expression by Eq. (6) should be used, that is indicated by the superscript LO.

The generalization of Eq.10b is somewhat more complex. We use Eqs. (11) and (10b) for the LO structure functions in order to find

$$
\begin{gathered}
x F_{3}^{Z}-C_{3}^{0} x F_{3}^{W}=-C_{3}^{1}\left(\Delta F_{2}^{W(\mathrm{LO})}+\right. \\
\left.\int_{x}^{1} \mathrm{~d} z K_{3}\left(z, \alpha_{S}\right) \Delta F_{2}^{W(\mathrm{LO})}\left(\frac{x}{z}, Q^{2}\right)\right),
\end{gathered}
$$

where, similar to Eq.112a), from the left we have the full structure functions and from the right the parton-model expression should be used.

Equations (10a) and 10b [as well as $\alpha_{S}$-corrected Eqs. [12a and [12b] hold for a generic target, not necessarily isoscalar. We note that strong interaction corrections come through the isosvector PDF and/or $s$ or $c$ quark distribution. For an isoscalar target the isovector distributions vanish $F^{(1, \pm)}=0$, provided that the isospin symmetry is exact. If we further neglect the contributions from $s$ and $c$ quarks, then the asymmetries $\Delta F_{2,3}$ vanish and Eqs. (12) reduce to the LS and PW relationships for the structure functions

$F_{2}^{Z} / F_{2}^{W}=C_{2}^{0}$,
$F_{3}^{Z} / F_{3}^{W}=C_{3}^{0}$.

Relationships (11) and (2) follow if we additionaly neglect contributions from the longitudinal structure function. It must be remarked, however, that the accuracy of the relations in the $C$-odd channel is higher, because they involve asymmetries $s-\bar{s}$ and $c-\bar{c}$, while the $C$-even relations are strongly corrected by strange quark effect at small $x$. Note also that if $s=\bar{s}$ and $c=\bar{c}$ (which is widely employed assumption in PDF analyses) then $\Delta F_{2, L}^{W}=0$ and Eqs. 13b) and (11) hold to all orders in $\alpha_{S}$ for the isoscalar target, as it follows from Eq. (12b). We also note that $\mathcal{O}\left(\alpha_{S}\right)$ corrections for the $\mathrm{NC} / \mathrm{CC}$ ratios of (anti)neutrino cross sections for the isoscalar target were recently discussed in Ref. 13, in which it was argued that the $\alpha_{S}$ correction is suppressed by a factor of $\sin ^{4} \theta_{W}$. For the full analysis one has to take into account electro-weak corrections as well 14 15.

If the target is not isoscalar then isovector quark distributions $F^{(1, \pm)}$ may be finite, which is the case for complex nuclei. The isovector quark distributions violate the LS and PW relationships for structure functions and cross sections. In order to address this effect, in Sect. 3 we discuss nuclear effects for the isoscalar and isovector quark distributions.

\section{Nuclear PDFs with isospin 0 and 1}

Complex nuclei, such as iron, have unequal number of neutrons $(N)$ and protons $(Z)$, and the 
isovector quark distribution is finite in such nuclei. In order to quantitatively understand this effect, we denote $q_{a / T}$ as the distribution of quarks of type $a$ in a target $T$. If $x$ is large enough to neglect coherent nuclear shadowing effect, the lepton scattering off a nucleus can be well approximated as incoherent scattering off bound protons and neutrons (for a recent review of nuclear deeply inelastic scattering see [16]). A widely used approximation is to neglect final state interactions of produced hadronic states with recoiling nucleus. In this approximation the nuclear parton distribuitions (nPDF) can be written as

$q_{a / A}=\left\langle q_{a / p}\right\rangle_{p}+\left\langle q_{a / n}\right\rangle_{n}$,

where the two terms in the right side are the quark distributions in bound protons and neutrons averaged with the proton and neutron nuclear spectral functions, respectively. Similar equations can also be written for antiquark distributions. The explicit expression for the averaging in Eq. (14) is (for derivation and more details see 172023 )

$$
\begin{gathered}
x\left\langle q_{a / p}\right\rangle_{p}=\int \mathrm{d} \varepsilon \mathrm{d}^{3} \boldsymbol{k} \mathcal{P}_{p}(\varepsilon, \boldsymbol{k})\left(1+k_{z} / M\right) \\
x^{\prime} q_{a / p}\left(x^{\prime}, Q^{2}, k^{2}\right), \\
x^{\prime}=\frac{Q^{2}}{2 k \cdot q}=\frac{x}{1+\left(\varepsilon+k_{z}\right) / M} .
\end{gathered}
$$

The integration in Eq.(15) is taken over the energy and momentum of bound protons (we separate the nucleon mass $M$ from the nucleon energy $\left.k_{0}=M+\varepsilon\right)$. The quantity $\mathcal{P}_{p}(\varepsilon, \boldsymbol{k})$ is the nuclear spectral function which describes the distribution of bound protons over the energy and momentum in nuclear ground state. The spectral functions $\mathcal{P}_{p}$ and $\mathcal{P}_{n}$ are normalized to the proton and neutron number, respectively. In Eq. (15), the $z$-axis is chosen in the direction opposite to the momentum transfer $q=\left(q_{0}, 0_{\perp},-|\boldsymbol{q}|\right)$, and $x^{\prime}$ is the Bjorken variable of the bound proton with four-momentum $k$. Since bound nucleons are off-mass-shell particles their quark distributions generally depend on nucleon virtuality $k^{2}$ as additional variable (off-shell effect in structure functions is discussed in terms of different ap- proaches in 181920212223). Equation similar to Eq. (15) also holds for neutrons with the obvious replacement of the spectral function and quark distributions.

Equations (14) and (15) account for nuclear binding and Fermi motion effects in nPDF (for this effect we will use the abbreviation FMB). For the isoscalar and isovector nuclear parton distributions we obtain from Eq. (14)

$q_{0 / A}=\left\langle q_{0 / p}\right\rangle_{0}$,

$q_{1 / A}=\left\langle q_{1 / p}\right\rangle_{1}$,

where the averaging is respectively performed with isoscalar and isovector spectral functions, $\mathcal{P}_{0}=\mathcal{P}_{p}+\mathcal{P}_{n}$ and $\mathcal{P}_{1}=\mathcal{P}_{p}-\mathcal{P}_{n}$.

The isoscalar and isovector spectral functions $\mathcal{P}_{0}$ and $\mathcal{P}_{1}$ are very different in complex nuclei. In an isoscalar nucleus with equal number of protons and neutrons one generally assumes vanishing $\mathcal{P}_{1}$ and nuclear effects are dominated by the isoscalar spectral function. In a nuclear mean-field model, in which a nucleus is viewed as Fermi gas of nucleons bound to self-consistent mean field, the spectral function can be calculated as

$\mathcal{P}_{\mathrm{MF}}(\varepsilon, \boldsymbol{p})=\sum_{\lambda<\lambda_{F}} n_{\lambda}\left|\phi_{\lambda}(\boldsymbol{p})\right|^{2} \delta\left(\varepsilon-\varepsilon_{\lambda}\right)$,

where $\phi_{\lambda}(\boldsymbol{p})$ is the wave function of the singleparticle level $\lambda$ in nuclear mean field and $n_{\lambda}$ is the number of nucleons on this level. The sum in Eq.(18) runs over occupied single-particle levels with energies below the Fermi level $\lambda_{F}$. Equation (18) gives a good approximation to nuclear spectral function in the vicinity of the Fermi level, where the excitation energies of the residual nucleus are small. As the separation energy $|\varepsilon|$ becomes higher, the mean-field approximation becomes less accurate. High-energy and highmomentum component of nuclear spectrum can not be described by the mean-field model and driven by correlation effects in nuclear ground state as witnessed by numerous studies (see, e.g., [24 25]). We denote this contribution to the spectral function as $\mathcal{P}_{\text {cor }}(\varepsilon, \boldsymbol{p})$.

For a generic nucleus the spectral function $\mathcal{P}_{1}$ determines the isovector nucleon distribution. We 
now argue that the strength of $\mathcal{P}_{1}$ for complex nuclei is peaked about the Fermi surface. It is reasonable to assume that $\mathcal{P}_{\text {cor }}$ is mainly isoscalar and neglect its contribution to $\mathcal{P}_{1}$. Then $\mathcal{P}_{1}$ is determined by the difference of the proton and neutron mean-field spectral functions. If we further neglect small differences between the energy levels of protons and neutrons then $\mathcal{P}_{1}$ will be determined by the difference in the level occupation numbers $n_{\lambda}$ for protons and neutrons. Because of Pauli principle, an additional particle can join a Fermi system only on an unoccupied level. In a complex nucleus all but the Fermi level are usually occupied (the Fermi level has a large degeneracy factor). Therefore, $\mathcal{P}_{1}$ is determined by the contribution from the Fermi level and we can write

$\mathcal{P}_{1}=(Z-N)\left|\phi_{F}(\boldsymbol{p})\right|^{2} \delta\left(\varepsilon-\varepsilon_{F}\right)$,

where $\varepsilon_{F}$ and $\phi_{F}$ are the energy and the wave function of the Fermi level.

Figure 1illustrates isospin dependence of nuclear effects for valence quark distributions. Shown are the ratios $R_{0}^{A}=F_{A}^{(0,-)} /\left(A F_{p}^{(0,-)}\right)$ and $R_{1}^{A}=$ $F_{A}^{(1,-)} /\left[(Z-N) F_{p}^{(1,-)}\right]$ calculated for the nucleus ${ }^{56} \mathrm{Fe}_{26}$. For the isoscalar nuclear spectral function we use the model spectral function of Ref. 25] (see also [26] for a different way of fixing parameters of the spectral function) which takes into account both the mean-field and correlated contributions. The isovector spectral function was calculated by Eq. (19) using the Fermi gas model in which the wave function of the Fermi level $\left|\phi_{F}(p)\right|^{2}=\delta\left(p-p_{F}\right) /\left(4 \pi p_{F}^{2}\right)$, where $p_{F}$ is the Fermi momentum. In numerical estimates we use $\varepsilon_{F}=-10 \mathrm{MeV}$ and $p_{F}=260 \mathrm{MeV}$ for the Fermi energy and momentum.

As an application of the present analysis we calculate the isovector correction to relation 13b. Using Eq. 12b and neglecting $\alpha_{S}$ correction we obtain for the ratio of $\mathrm{NC}$ and $\mathrm{CC}$ nuclear structure functions

$\frac{F_{3}^{Z}}{F_{3}^{W}}=1-\sin ^{2} \theta_{W}\left(2-\frac{2}{3} \delta N R_{1 / 0}^{A}(x)\right)$,

where $\delta N=(N-Z) / A$ is fractional neutron excess in a nucleus and $R_{1 / 0}^{A}$ is the ratio of re-

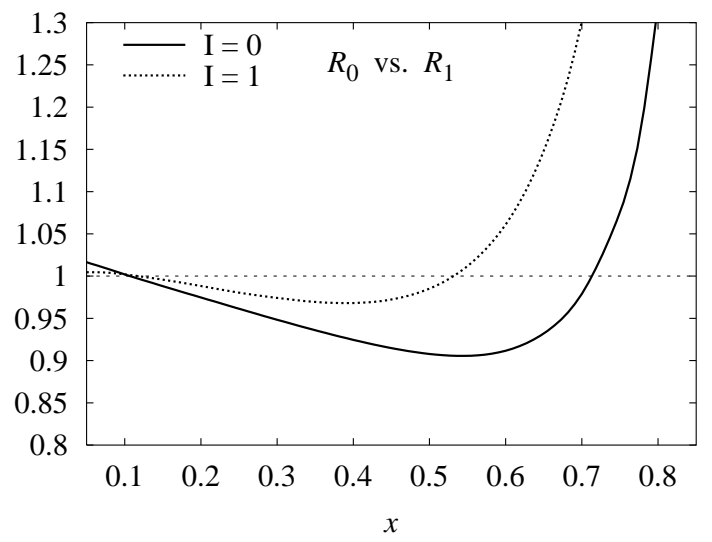

Figure 1. Fermi motion and nuclear binding effects for isoscalar and isovector quark distributions in ${ }^{56} \mathrm{Fe}_{26}$ calculated in impulse approximation for $Q^{2}=15 \mathrm{GeV}^{2}$.

duced nuclear isovector and isoscalar quark distributions, $\left[F_{A}^{(1,-)} /(Z-N)\right] /\left(F_{A}^{(0,-)} / A\right)$. The latter can also be written in terms of the corresponding ratio for the proton $R_{1 / 0}^{p}=F_{p}^{(1,-)} / F_{p}^{(0,-)}$ and the ratios $R_{1}$ and $R_{0}$ as $R_{1 / 0}^{A}=R_{1 / 0}^{p} R_{1}^{A} / R_{0}^{A}$. Figure 2 shows the $x$ dependence of $R_{1 / 0}^{A}$ for the iron nucleus and the ratio $R_{1 / 0}^{p}$ for the proton.

The parameter controlling the isovector correction in Eq. (20) is $\delta N$. This correction is additionally suppressed by the factor of $\sin ^{2} \theta_{W}$. Given that $\delta N \approx 0.07$ for iron we observe that the isovector correction in Eq.(20) is not large. In particular, in the valence quark region of $x \sim 0.3$ the magnitude of the correction in Eq. (20) is about $1 \%$ of the $\mathrm{PW}$ value $1-2 \sin ^{2} \theta_{W}$. This correction rises with $x$, as is clear from Fig. 2 However, its magnitude is only about $2 \%$ at $x=0.7$.

We note that the non-isoscalarity correction to $R^{-}$for the total cross sections was recently discussed in 2728 . It was observed that the proper treatment of the FMB effect leads to about $6 \%$ enhancement of the neutron excess correction for iron nucleus. We also remark that a subtle cancellation of perturbative QCD effects in the isovector 


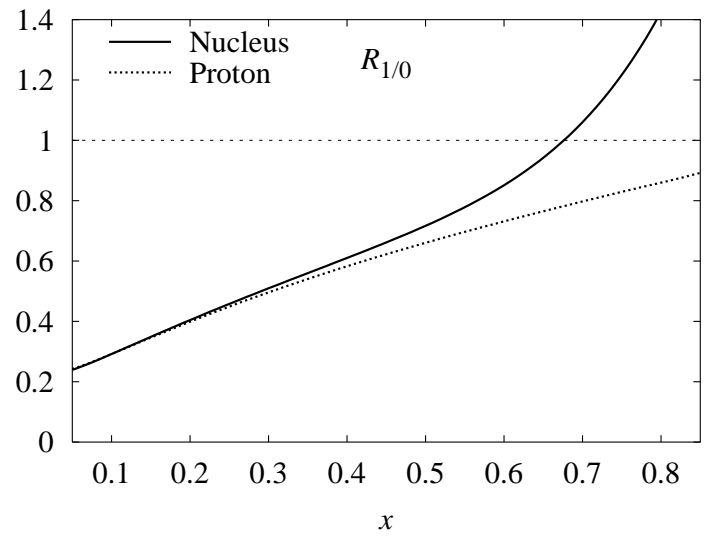

Figure 2. The ratio of isovector and isoscalar quark distributions calculated for the proton and iron at $Q^{2}=15 \mathrm{GeV}^{2}$.

correction for $R^{-}$was found in 28 .

In summary, we discussed relations between $\mathrm{NC}$ and $\mathrm{CC}$ structure functions for a generic nuclear target. We argued that the relation $F_{3}^{Z} / F_{3}^{W}=1-2 \sin ^{2} \theta_{W}$ survives strong interaction corrections if the strange sea in the target is symmetric and the target is isoscalar. The corresponding relation for $F_{2}$ is less accurate and affected by strange quark effect at small $x$. We also discussed how the parton distributions with isospin 0 and 1 change in complex nuclei and applied the results to compute the non-isoscalarity correction to the ratio $F_{3}^{Z} / F_{3}^{W}$.

\section{Acknowledgments}

This work was partially supported by the Russian Foundation for Basic Research projects no. 03-02-17177. I am grateful to the organizers of the NuInt04 meeting for local support.

\section{REFERENCES}

1. Proceedings of Conferences of NuInt series.

2. M. L. Mangano, et al., arXiv: hep$\mathrm{ph} / 0105155$.
3. Minerva Collaboration (D. Drakoulakos et al.), arXiv: hep-ex/0405002.

4. G. P. Zeller, et al., Phys. Rev. Lett. 88, 091802 (2002).

5. E. A. Paschos and L. Wolfenstein, Phys. Rev. D 7, 91 (1973).

6. C. H. Llewellyn-Smith, Nucl. Phys. B 228, 205 (1983).

7. S. Davidson, S. Forte, P. Gambino, N. Rius, and A. Strumia, JHEP 02, 037 (2002).

8. K. S. McFarland and S.-O. Moch, arXiv: hepph/0306052.

9. S. Kretzer, F. Olness, J. Pumplin, D. Stump, W. K. Tung and M. H. Reno, Phys. Rev. Lett. 93, 041802 (2004).

10. B. Portheault, arXiv:hep-ph/0406226

11. J. T. Londergan and A. W. Thomas, Phys. Lett. B 558, 132 (2003).

12. G. Altarelli, R. K. Ellis, and G. Martinelli, Nucl. Phys. B 143, 521 (1978); Erratum-ibid. B146, 544 (1978).

13. B. A. Dobrescu and R. K. Ellis, Phys. Rev. D 69, 114014 (2004).

14. K. P. O. Diener, S. Dittmaier and W. Hollik, Phys. Rev. D 69, 073005 (2004).

15. A. B. Arbuzov, D. Y. Bardin and L. V. Kalinovskaya, arXiv:hep-ph/0407203

16. G. Piller and W. Weise, Phys. Rept. 330, 1 (2000).

17. S. A. Kulagin, Nucl. Phys. A 500, 653 (1989).

18. F. Gross and S. Liuti, Phys. Rev. C 45, 1374 (1992).

19. W. Melnitchouk, A. W. Schreiber and A. W. Thomas, Phys. Rev. D 49, 1183 (1994).

20. S. A. Kulagin, G. Piller, and W. Weise, Phys. Rev. C 50, 1154 (1994).

21. S. A. Kulagin, Nucl. Phys. A 640, 435 (1998).

22. S. I. Alekhin, S. A. Kulagin and S. Liuti, Phys. Rev. D 69, 114009 (2004).

23. S. A. Kulagin and R. Petti, paper in preparation.

24. O. Benhar, A. Fabrocini, S. Fantoni, and I. Sick, Nucl. Phys. A 579, 493 (1994).

25. C. Ciofi degli Atti and S. Simula, Phys. Rev. C 53, 1689 (1996).

26. S. A. Kulagin and A. V. Sidorov, Eur. Phys. J. A 9, 261 (2000).

27. S. A. Kulagin, Phys. Rev. D 67, 091301(R) 
(2003).

28. S. A. Kulagin, arXiv: hep-ph/0406220. 